\title{
Sincronização folicular e vascularização do folículo dominante em novilhas mestiças tratadas com estradiol*
}

\section{Follicle synchronization and changes in vascularization of the dominant follicle in crossbred heifers after estradiol treatment}

\author{
Miller Pereira Palhão, ${ }^{* *}$ Cláudio do Santos Piedade, ${ }^{* *}$ Henrique Lentulo Araújo, ${ }^{* * *}$ Carlos Antônio de Carvalho Fernandes, ${ }^{* *}$ \\ Cristiano Rodrigues Borges Guimarães, ${ }^{* * *}$ Josiane Rossi Ribeiro, ${ }^{* *}$ Willian de Souza Matias Reis, ${ }^{* * * *}$ \\ João Henrique Moreira Viana**,*****
}

\begin{abstract}
Resumo
Avaliou-se a sincronização folicular e vascularização na parede do folículo dominante (FD) após o tratamento com Cipionato (CE) ou Benzoato de estradiol (BE). No Experimento 1, 45 novilhas receberam implante de $P_{4}(1,0 \mathrm{~g})$ e foram distribuídas em 3 grupos de acordo com éster de estradiol administrado pela via intramuscular: $1 \mathrm{~mL}$ salina; $0,5 \mathrm{mg} \mathrm{CE}$ ou 2,0mg BE. O diâmetro do FD e a emergência folicular (EF) foram monitorados por 5 dias. No experimento 2, D0 (dia 0): 30 novilhas receberam implante de $\mathrm{P}_{4}$ e 2,0mg BE; D8, retirada do implante e 0,5mg de prostaglandina (cloprostenol); D9: tratamentos: $1 \mathrm{~mL}$ salina; 0,5mg CE ou $1,0 \mathrm{mg}$ BE. O diâmetro e a vascularização do FD foram monitorados do D8 ao D10. Os dados foram analisados por ANOVA ou Kruskal-Wallis, e as médias comparadas por Tukey (5\%). No experimento 1, a EF ocorreu mais tarde $(P<0,0013)$ no grupo BE $(4,3 \pm 0,8$ vs. $3,5 \pm 0,8 d)$. No experimento 2 , o fluxo sanguíneo do FD aumentou $(P<0,001) 24 \mathrm{~h}$ após o tratamento com $B E$. A taxa de ovulação foi semelhante $(P>0,05)$ entre os grupos $(80,0,70,0$ e $55,5 \%$, BE, CE e salina). Conclui-se que nas concentrações utilizadas, o BE, no início do protocolo, retardou a EF e, no final do protocolo, promoveu uma melhor sincronia do FD demonstrada pela elevação do fluxo sanguíneo.
\end{abstract}

Palavras-chave: fisiologia da reprodução; protocolo hormonal; Doppler colorido; ovulação.

\begin{abstract}
The study aimed to evaluate synchronization and blood flow of the dominant follicle (DF) after estradiol cypionate (EC) or benzoate (EB) treatment. In experiment 1, progesterone (P4) implants $(1.0 \mathrm{~g})$ were inserted in 45 heifers, which were given $1 \mathrm{~mL}$ saline, $0.5 \mathrm{mg}$ EC or 2.0mg EB. The diameter of the DF and follicle emergence (FE) were recorded for 5 days. For experiment 2, 30 heifers were given: D0 (day 0): P4 implant and 2.0mg EB; D8: implant removal and 0.5mg of prostaglandin (cloprostenol). On D9, they were assigned in $1 \mathrm{~mL}$ saline, $0.5 \mathrm{mg}$ EC or $1.0 \mathrm{mg}$ EB. From D8 to D10, the diameter and blood flow of the DF was monitored. Data were analyzed by ANOVA or Kruskal-Wallis test, and compared by Tukey (5\%). In experiment 1 , the FE occurred later $(P<0.0013)$ in EB group (4.3 \pm 0.8 vs. 3.5 $\pm 0.8 \mathrm{~d})$. In experiment 2, blood flow of the DF increased $(P<0.001) 24 \mathrm{~h}$ after EB treatment. The ovulation rate was similar $(P>0.05)$ between groups $(80.0,70.0$ and $55.5 \%, E B$, EC and saline). The interpretation was that, in these concentrations, the EB, at the beginning, delayed FE and, at the end of the protocol, promoted a better synchrony of the $\mathrm{DF}$, confirmed by the increase of the blood flow.
\end{abstract}

Keywords: physiology of reproduction; hormone protocol; color Doppler; ovulation.

\section{Introdução}

O advento de protocolos hormonais que permitem a inseminação artificial (IA) ou a transferência de embrião (TE) em momento previamente definido (IATF e TETF, respectivamente) permitiu ampla disseminação destas biotecnologias.

Dentro destes protocolos, os ésteres de estradiol possuem ação indireta sobre a função ovariana Adams, 1994). Quando administrados em animais com altas concentrações plasmáticas de progesterona (P4), atuam na hipófise, bloqueando seletivamente a produção de $\mathrm{FSH}$, induzindo atresia folicular e, posterior sincronia do desenvolvimento de uma nova onda folicular (Bigelow \& Fortune, 1998). Por outro lado, quando aplicados em fêmeas com baixa P4 plasmática, o hipotálamo responde com liberação de $\mathrm{GnRH}$, que age na hipófise, estimulando a liberação do pico ovulatório de LH (Fortune, 1994).

No Brasil, a legislação não faz restrições ao uso de derivados de estradiol em bovinos, onde eles são utilizados no início do protocolo para sincronizar a onda de crescimento folicular e ao final com a finalidade de induzir a ovulação (Martinez et al., 2002). Dentre os ésteres mais usados em protocolos hormonais, o Benzoato (BE) tem a meia vida mais curta, cerca

${ }^{*}$ Recebido em 16 de maio de 2014 e aceito em 30 de junho de 2014.

**Professor da Universidade José do Rosário Vellano - UNIFENAS, Rod. MG 179, Km 0, Campus Universitário, CEP: 37130-000, Alfenas, MG.

***Discente do Programa de Pós-graduação em Reprodução, Sanidade e Bem-estar Animal - UNIFENAS, Rod. MG 179, Km 0, Campus Universitário, CEP: $37130-000$, Alfenas, MG.

****Estudante da Graduação de Medicina Veterinária - UNIFENAS, Rod. MG 179, Km 0, Campus Universitário, CEP: 37130-000, Alfenas, MG

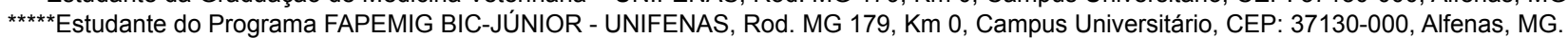

******Pesquisador da Embrapa Gado de Leite, Rua Eugênio do Nascimento, 610 - Dom Bosco, CEP 36038-330, Juiz de Fora, MG. Autor para correspondência: millerpalhao@yahoo.com.br 
de 3 dias, o valerato com meia vida de 7 a 9 dias e o Cipionato (CE) de 10 a 12 dias. Como indutor da ovulação, o CE é uma alternativa que possibilita a realização de protocolos de IATF e TETF com apenas três períodos de manejo. Permanecendo biodisponível de forma sistêmica por maior tempo, o CE pode ser administrado juntamente com a retirada do implante de progesterona, mantendo níveis plasmáticos suficientes para estimular o pico de LH após a queda dos níveis de progesterona (P4). Por outro lado, o BE, por apresentar uma farmacocinética que permite uma biodisponibilidade mais rápida e uma meia vida mais curta, é normalmente administrado em protocolos que exigem um manejo a mais, sendo aplicado $24 \mathrm{~h}$ após a retirada do dispositivo com progestágeno (Martinez et al., 2002).

O uso da ultrassonografia em modo B acoplada ao Doppler colorido permitiu a visualização de sinais de fluxo sanguíneo da parede do folículo durante seu desenvolvimento. Com a utilização desta técnica, é possível acessar as alterações de fluxo sanguíneo associadas à angiogênese perifolicular e à regressão da vascularização durante o desenvolvimento e à atresia folicular, respectivamente (Acosta \& Miyamoto, 2004).

Neste contexto, este estudo foi desenvolvido para acessar o padrão de regressão do folículo dominante e emergência folicular, após a administração de diferentes ésteres de estradiol no início de um protocolo hormonal. Também foram avaliados o desenvolvimento e a vascularização do folículo dominante antes e após a administração destes ésteres como indutor ovulatório.

\section{Material e métodos}

\section{Localização e animais}

O estudo foi conduzido na região Sul do estado de Minas Gerais e incluiu novilhas mestiças (Bos taurus taurus $x$ Bos taurus indicus) entre 23 a 36 meses, com peso entre 347 a $580 \mathrm{~kg}$, em bom estado clínico e nutricional (escore de condição corporal entre 3,0 e 3,5 ). Os animais foram previamente examinados por ultrassonografia para avaliar a função ovariana (probe de 7.5 MHz - M5 Mindray) e, mantidos em pastagem de Brachiaria brizantha com suplementação mineral e água à vontade. Todos os procedimentos animais contidos neste estudo foram aprovados pela Comissão de ética no uso de animais - CEUA - da UNIFENAS (Parecer N.o 48A/2011).

\section{Experimentos realizados}

Foram conduzidos dois experimentos utilizando um protocolo hormonal empregado para receptoras de embrião em programas de TETF. No início do protocolo (D0 - dia 0), fez-se a colocação de um dispositivo vaginal de progesterona (P4 - 1,0g, Primer $^{\circledR}$, Tecnopec, São Paulo, Brasil) e administração do éster de estradiol para sincronizar a onda de crescimento folicular. No D8, procedeu-se a retirada do dispositivo vaginal juntamente com a aplicação de prostaglandina (0.500mg de Cloprostenol Sódico, Sincrosin - Vallée, São Paulo, Brasil). No D9, uma nova dose de éster de estradiol foi administrada como indutor ovulatório. A previsão é de que as ovulações se concentrassem no D11.

\section{Experimento 1}

No D0 do protocolo hormonal, após o exame ultrassonográfico, as novilhas $(n=45)$ foram randomizadas, de acordo com o diâmetro do folículo dominante, em três grupos: Salina $(n=15)$ recebeu $1 \mathrm{~mL}$ de salina; CE $(n=15)-0,5 \mathrm{mg}$ de CE $(1 \mathrm{~mL}$, Cipionato de Estradiol Von Franken ${ }^{\circledR}$, Von Franken, Buenos Aires, Argentina) e BE $(n=15)$ - $2 m g$ de BE $\left(2 m L\right.$, Ric $B E^{\circledR}$, Tecnopec, São Paulo, Brasil). Demais procedimentos conforme o protocolo descrito anteriormente. Antes do tratamento, o diâmetro médio do FD não diferiu $(P>0,1)$ entre os grupos, medindo 9,9 $(6,2-12,6 \mathrm{~mm})$, $10,1(5,3-12,4 \mathrm{~mm})$ e $9,6 \mathrm{~mm}(6,5-13,0 \mathrm{~mm})$, respectivamente para salina, CE e BE.

Duas variáveis foram avaliadas: 1) regressão do folículo dominante e 2) intervalo do início do protocolo à emergência de uma nova onda folicular. Para tanto, os animais foram examinados no D0, e diariamente do D2 ao D5. Quando presente, o folículo dominante foi monitorado até o D5 e considerou-se como emergência folicular a primeira observação de um folículo em crescimento com diâmetro igual ou superior a $4,0 \mathrm{~mm}$.

\section{Experimento 2}

Foram utilizadas trinta $(n=30)$ novilhas mestiças. No D0, estes animais receberam um implante de P4 $(1,0 \mathrm{~g})$ e $2,0 \mathrm{mg}$ $(2 \mathrm{~mL})$ de BE para sincronizar a onda folicular. No D8, o FD foi mensurado, e os animais foram selecionados de acordo com o diâmetro do FD, para receber, no D9, um dos tratamentos para indução da ovulação: $1 \mathrm{~mL}$ Salina $(n=10)$; CE $(n=10)-0.5 \mathrm{mg}$ de CE; e BE $(n=10)$ - $1 \mathrm{mg} \mathrm{BE}$. Antes do tratamento, o diâmetro médio do FD não diferiu $(P>0,1)$ entre os grupos, medindo 8,8 $\mathrm{mm}(6,4-12,2 \mathrm{~mm}), 9,5(6,8-12,1 \mathrm{~mm})$ e $9,3 \mathrm{~mm}(7,4-12,7 \mathrm{~mm})$, respectivamente para salina, $\mathrm{CE}$ e $\mathrm{BE}$.

A cada exame, os ovários dos animais foram examinados pela ultrassonografia em módulo B para mensuração do diâmetro folicular. $O$ diâmetro de folículos não esféricos foi estimado usando a equação: $D=(A+B) / 2$, onde $A$ representa a máxima altura e B o máximo diâmetro transverso. A irrigação sanguínea presente na parede do folículo dominante foi avaliada na imagem gerada em módulo Doppler colorido. Para este último, a cada exame, foram salvos cerca de 100 quadros, contendo todas as seções transversais do FD. O quadro com o maior sinal de fluxo sanguíneo foi selecionado e a área da parede folicular contendo pixels coloridos foi medida com auxílio do software ImageJ (Processamento e Análise de Imagem em Java).

A metodologia de análise de imagens ultrassonográficas foi adaptada de Ginther (2007). Os escores foram estabelecidos entre 1 a 4, sendo o escore 1 dado a folículos com vascularização discreta ( $\leq 25 \%$ da parede com sinais coloridos), 2 - intermediária baixa (>25 e $\leq 50 \%), 3$ - intermediária alta (>50 e < $<5 \%$ ) e 4 vascularização intensa (>75\%).

Todos os exames ultrassonográficos foram realizados por um mesmo operador utilizando o aparelho (M5-Mindray) acoplado a um transdutor de $7.5 \mathrm{MHz}$. O aparelho foi predefinido com as configurações de modo-B em $6,5 \mathrm{MHz}$, com velocidade de 32 frames por segundo, ganho de 72 , potência de $100 \%$, definindo uma profundidade de trabalho de $6,1 \mathrm{~cm}$ e ponto focal distante $2 \mathrm{~cm}$ do transdutor. No modo-Doppler colorido, a configuração foi ajustada para uma escala de velocidades de $6,0 \mathrm{~cm} / \mathrm{s}$ com objetivo de detectar movimentos de hemácias em pequenos vasos ovarianos (Ginther, 2007). Para tanto, utilizou-se a frequência de $4,2 \mathrm{MHz}$, com 15 frames por segundo, potência de $100 \%$, ganho de 58 e o ajuste da frequência de pulsos de $0,6 \mathrm{kHz}(\mathrm{PRF}=0,6)$.

\section{Análises estatísticas}

Todos os dados de diâmetro folicular $(\mathrm{mm})$ foram submetidos ao teste de Shapiro-Wilk para averiguar a normalidade. 
Posteriormente, o quadro da ANOVA para a regressão do FD foi preenchido pelos seguintes efeitos: Grupo (Salina, $0,5 \mathrm{mg}$ de CE e 2,0mg de BE), Dia e a Interação Grupo vs. Dia. As médias dos grupos foram comparadas em cada dia, ou dentro de cada grupo, as diferenças entre as médias dos dias também foram acessadas pelo teste de Tukey. Outras variáveis, como dia da emergência folicular e diâmetro do folículo à emergência e à ovulação, foram submetidas à ANOVA e o efeito principal de grupo foi avaliado. Dados qualitativos foram analisados pelo teste de Qui-quadrado. A variação dos escores de fluxo sanguíneo na parede do FD foi acessada pela estatística de Kruskal Wallis. As análises foram processadas no programa SAS (SAS Institute versão 9.3, USA), probabilidades $<5 \%$ foram consideradas significativas e aquelas entre 5 e $10 \%$ consideradas aproximações.

\section{Resultados}

\section{Experimento 1}

A Figura 2 mostra o efeito de grupo $(P<0,003)$ indicando que o diâmetro médio do FD, ao longo dos dias, foi menor $(P<0,05)$ no grupo tratado com BE em comparação aos demais. Nos dois primeiros dias, a regressão do FD divergiu $(P<0,07)$ entre os grupos $\mathrm{BE}(-1,0 \pm 0,4 \mathrm{~mm})$ e CE $(-0,2 \pm 0,2 \mathrm{~mm})$. Após o dia 2 , o comportamento do FD foi semelhante $(P<0,4)$ entre os grupos, com redução aproximada de 2,4 mm entre os dias 2 e 5. Entretanto, no grupo tratado com cipionato a redução foi significativa no dia 4, fenômeno observado um dia mais tarde no grupo BE (dia 5). No grupo salina, o efeito de dia não foi significante $(P>0,05)$, e o diâmetro do FD permaneceu constante durante o período de avaliação (Fig.1).

A emergência folicular (EF) não foi observada em sete animais do grupo Salina, em dois tratados com CE e em dois com BE. $O$ dia de ocorrência da EF diferiu $(P<0,0013)$ entre os grupos tratados (Tab.1). Em ambos os grupos (CE e BE), a EF ocorreu imediatamente antes da redução significante no diâmetro do FD (Figura 1). Apesar das diferenças no dia de emergência, o diâmetro do novo folículo foi semelhante $(P>0,1)$ entre os grupos tratados com CE e BE (Tab.1). O percentual de animais que apresentaram EF nos cinco dias avaliados foi menor $(P<0,05)$ no grupo salina e o diâmetro do folículo neste momento foi maior $(6,0 \pm 1,2 \mathrm{~mm}, \mathrm{P}<0,005)$ em comparação aos demais grupos (Tab.1).

\section{Experimento 2}

Um animal do grupo Salina apresentou um crescimento folicular anormal (15,9mm no D8), valor maior que duas vezes o desvio padrão $(1,7 \mathrm{~mm})$ da média geral $(9,1 \mathrm{~mm})$. Os dados deste animal foram considerados "outliers" e não foram incluídos nas análises estatísticas $(n=29)$.

O efeito significativo de Dia $(P<0,001)$ evidencia o crescimento do FD entre o D8 e D9 (Figura 2). O crescimento do folículo não diferiu $(P>0,1)$ entre os grupos.

O fluxo sanguíneo na parede do FD variou somente em resposta ao BE (Figura 3), onde o fluxo sanguíneo aumentou $(\mathrm{P}<0,0006)$ 24 horas após a administração (entre D9 e D10). No grupo CE, apesar de significante $(P<0,008)$, a elevação foi perceptível somente entre D8 e D10. No grupo salina, o fluxo sanguíneo não diferiu $(P>0,1)$ ao longo dos dias avaliados.
No D10, o diâmetro médio do FD foi menor $(P<0,04)$ nos animais induzidos a ovular com $1,0 \mathrm{mg}$ de $\mathrm{BE}(11,4 \pm 0,5)$, comparando com o CE $(12,7 \pm 0,3 \mathrm{~mm})$ como mostra a Tabela 2 . O percentual de ovulação não diferiu $(P>0,1)$ entre os grupos.

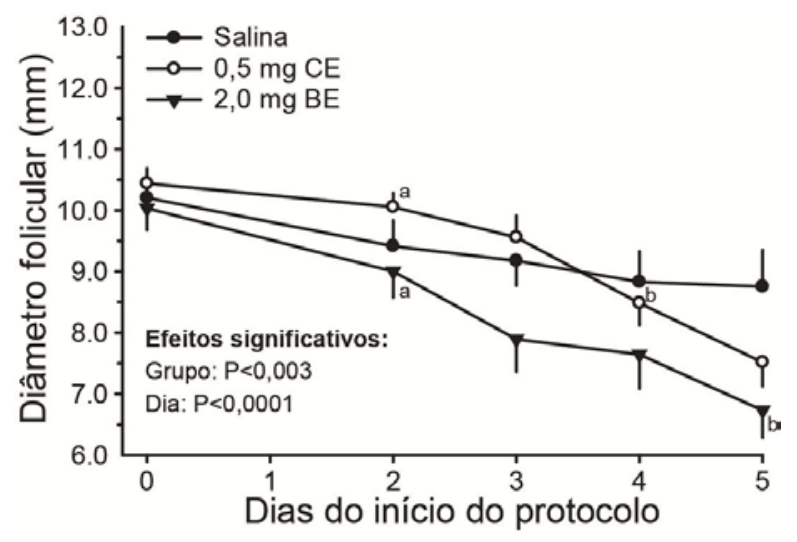

Figura 1: Efeito dos diferentes ésteres de estradiol sobre o folículo dominante presente no início do protocolo. O efeito de grupo $(P<0,003)$ indica diferença entre os animais tratados com BE e CE, e o efeito de Dia $(P<0,0001)$ demonstra a queda no diâmetro folicular nestes dois grupos. ${ }^{\mathrm{a}, \mathrm{b}}$ Letras diferentes dentro de um mesmo grupo indicam diferença significativa $(P<0,05)$

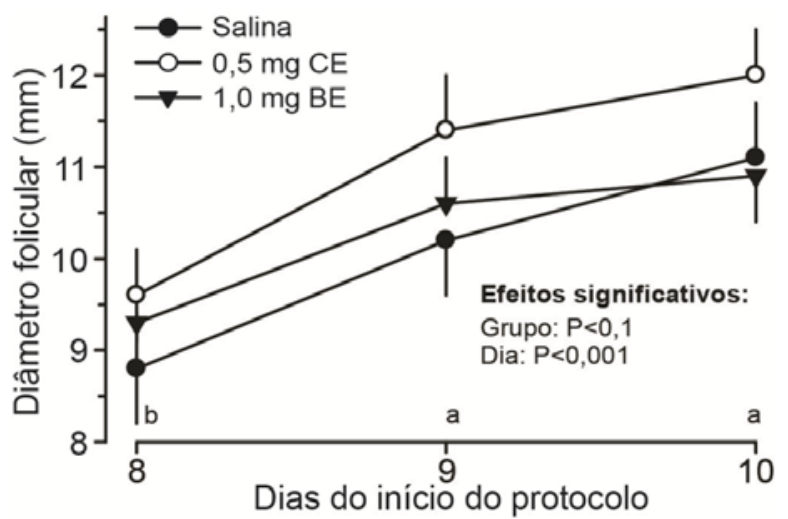

Figura 2: Diâmetro do folículo ovulatório em cada tratamento ao longo dos dias após o início do protocolo. O efeito significativo de $\mathrm{Dia}(\mathrm{P}<0,001)$ indica o crescimento folicular entre os dias 8 e 9 , observado em todos os tratamentos. a,b Letras diferentes indicam diferenças estatísticas entre os dias, dentro de cada grupo (Teste de Tukey, Salina: $\mathrm{P}<0,04 ; 0,5 \mathrm{mg} C E$ : $\mathrm{P}<0,01 ; 1 \mathrm{mg} \mathrm{BE}$ : $P<0,07$; e média geral: $P<0,001$ ).

Tabela 1: Emergência folicular (EF) e diâmetro do folículo dominante no momento da EF em novilhas mestiças (Bos taurus taurus $\times$ Bos taurus indicus) tratadas com Salina, CE e BE

\begin{tabular}{lccc}
\hline Grupo & $\begin{array}{c}\text { Animais com } \\
\text { EF (\%) }\end{array}$ & Dia da EF & $\begin{array}{c}\text { Diâmetro na } \\
\text { EF (mm) }\end{array}$ \\
\hline Salina & $8 / 15(53,3)$ & $2,8 \pm 0,8^{\mathrm{b}}$ & $6,0 \pm 1,2^{\mathrm{a}}$ \\
$\mathbf{0 , 5} \mathbf{~ m g ~ C E}$ & $13 / 15(86,7)$ & $3,5 \pm 0,8^{\mathrm{b}}$ & $5,0 \pm 0,9^{\mathrm{b}}$ \\
$\mathbf{2 , 0} \mathbf{~ m g ~ B E}$ & $13 / 15(86,7)$ & $4,3 \pm 0,8^{\mathrm{a}}$ & $4,6 \pm 0,4^{\mathrm{b}}$ \\
\hline
\end{tabular}

a,b Letras diferentes na coluna indicam diferenças significativa (5\% de probabilidade pelo Teste "Tukey"). 


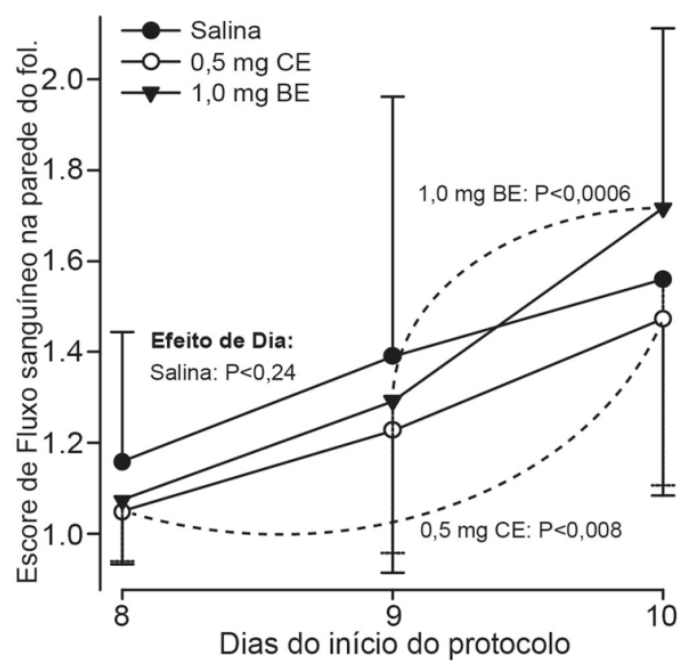

Figura 3: Fluxo sanguíneo (escores designados pela quantidade de sinais recebidos da vascularização) na parede do folículo dominante em cada tratamento ao longo dos dias após o início do protocolo. O efeito de dia foi significante nos grupos $C E(P<0,008)$ e $B E(P<0,0006)$ indicando aumento da vascularização (dias 8-10 e 9-10, respectivamente para CE e BE).

Tabela 2: Diâmetro do folículo pré-ovulatório e taxa de ovulação em novilhas mestiças (Bos taurus taurus x Bos taurus indicus) tratadas com Salina, CE e BE

\begin{tabular}{lcc}
\hline Grupo & Taxa de ovulação (\%) & $\begin{array}{c}\text { Diâmetro pré-ovulatório } \\
\text { ( } \mathbf{m m})\end{array}$ \\
\hline Salina & $5 / 9(55,5)$ & $12,6 \pm 0,8^{\mathrm{ab}}$ \\
$\mathbf{0 , 5} \mathbf{~ m g ~ C E}$ & $7 / 10(70,0)$ & $12,7 \pm 0,3^{\mathrm{a}}$ \\
$\mathbf{1 , 0} \mathbf{~ m g ~ B E}$ & $8 / 10(80,0)$ & $11,4 \pm 0,5^{\mathrm{b}}$ \\
\hline
\end{tabular}

$a, b$ Letras diferentes nas colunas indicam diferença significativa $(p<0,05)$.

\section{Discussão}

Os resultados deste estudo confirmam os efeitos dos ésteres de estradiol sobre as três etapas: regressão do folículo dominante, emergência de uma nova onda e desenvolvimento do folículo ovulatório.

A regressão folicular e a emergência da nova onda tiveram diferentes comportamentos de acordo com o princípio utilizado. Nos dois primeiros dias após o tratamento, a redução no diâmetro folicular foi mais efetiva no grupo BE. No entanto, este contraste inicial não parece ter efeito direto sobre a emergência folicular. O mais importante neste caso parece ser a queda significativa no diâmetro do folículo dominante, que ocorreu mais tarde no grupo tratado com BE.

É importante ter cautela para interpretar esses dados, uma vez que o estradiol utilizado para regredir o FD no início do protocolo também atua como supressor de $\mathrm{FSH}$, que atua no desenvolvimento da nova onda folicular (PALHAO et al., 2009).
Dessa forma, tanto a queda do estradiol endógeno quanto a do exógeno (tratamento) devem ser consideradas para o melhor entendimento do fenômeno de emergência folicular.

A emergência folicular parece ser dependente da dose de estradiol, uma vez que 1 ou $5 \mathrm{mg}$ de BE promoveram o aparecimento de uma nova onda folicular 3,6 ou 5,5 d após a administração (Martinez et al., 2005). Estes dados suportam o observado neste estudo, onde a utilização de $2 \mathrm{mg}$ de $B E$ resultou em um intervalo intermediário (4,5 d) entre a aplicação e a emergência folicular. Neste caso, o efeito inibitório do estradiol - dose dependente (Palhao et al., 2010) - sobre o FSH controlaria o momento da emergência folicular. Por outro lado, o que chama atenção neste estudo foi a observação da emergência folicular relacionada a redução significativa no diâmetro do FD, podendo ser um indicativo da perda de funcionalidade desta estrutura. Nestes termos, a emergência folicular ocorrida 1 dia mais cedo no grupo CE se explicaria pela menor dose $(0,5 \mathrm{mg})$ utilizada e, uma possível ação mais efetiva deste princípio sobre a funcionalidade do FD.

No experimento 2 era esperado que o crescimento do folículo dominante fosse semelhante entre os grupos, devido a intervenção ocorrida somente na indução ovulatória. Nesta etapa final de desenvolvimento, a proliferação da vascularização coincide com seu crescimento do FD, o que sugere que o processo de seleção esteja associado à formação de um maior plexo vascular (Acosta, 2007). Nestes termos, uma classificação do folículo dominante segundo sua vascularização poderia indicar maiores chances deste folículo alcançar a maturação final e adquirir a capacidade ovulatória. No entanto, neste estudo, o exame ultrassonográfico da vascularização não permitiu nenhuma inferência sobre a capacidade ovulatória dos folículos observados. Talvez a realização de avaliações seriadas, assim como feita por Siddiqui et al. (2009), possa fornecer informações mais completas do aumento ou da diminuição da vascularização folicular e, assim estabelecer uma diferença entre dominância e atresia.

No período periovulatório, no entanto, ocorre ruptura da membrana basal do folículo e uma penetração de vasos sanguíneos intrafolicular, o que contribui para um aumento no sinal de Doppler captado nestes folículos (Campbell et al., 2003). Neste estudo foi possível perceber uma elevação no fluxo sanguíneo, na parede do folículo dominante, $24 \mathrm{~h}$ após o tratamento com BE. Como o pico de LH causa elevação do fluxo sanguíneo na parede do folículo ovulatório (Acosta, 2007), é possível correlacionar este fato com uma maior sincronia dos animais tratados com BE como indutor ovulatório. Outra evidência da maior sincronia dos animais neste grupo foi o menor diâmetro do folículo ovulatório, uma vez que o crescimento folicular se reduz após o pico de LH (Palhao et al., 2009).

Conclui-se que o benzoato de estradiol retarda a emergência folicular e eleva o fluxo sanguíneo na parede do folículo dominante, melhorando a sincronia da ovulação. A ultrassonografia em Doppler colorido é um bom indicador da proximidade do pico de LH e possibilita a avaliação da eficiência de protocolos hormonais.

\section{Agradecimentos}

Os autores agradecem à Fundação de Amparo à Pesquisa do Estado de Minas Gerais (Fapemig - projeto CVC-APQ 01454/12); ao Conselho Nacional de Pesquisa (CNPq), pelo suporte financeiro; às empresas Vallée S.A. e Biotran Assessoria e Consultoria em Medicina Veterinária Ltda., pelo apoio financeiro e logístico para a realização do trabalho. 


\section{Referências}

ADAMS, G.P. Control of ovarian follicular wave dynamics in cattle: implications for synchronization and superstimulation. Theriogenology. v. 41, p. 19-24, 1994.

BIGELOW, K.L.; FORTUNE, J.E. Characteristics of prolonged dominant versus control follicles: follicle cell numbers, steroidogenic capabilities and messenger ribonucleic acid for steroidogenic enzymes. Biology of Reproduction. v. 58, p. 12411249. 1998.

FORTUNE, J.E. Ovarian follicular growth and development in mammals. Biology of Reproduction, v. 50, p. 225-232, 1994.

ACOSTA, T.J. \& MIYAMOTO, A. Vascular control of ovarian function: ovulation, corpus luteum formation and regression. Animal Reproduction Science. v. 82-83, p. 127-140, 2004.

ACOSTA, T.J. Studies of follicular vascularity associated with follicle selection and ovulation in cattle - Review. Journal of Reproduction and Development. v. 53, p. 39-44, 2007.

CAMPBELL, S., BOURNE, T.H., WATERSTONE, J. Transvaginal colour blood flow imaging of the periovulatory follicle. Fertility and Sterility. v. 60, p. 433-438, 2003.

GINTHER, O.J. Follicle blood flow. In Ultrasonic Imaging and Animal Reproduction: Color-Doppler Ultrasonography. $1^{\text {st }}$ Edition, Equiservises Publishing. Chapter 5, p.87-114, 2007.
MARTINEZ, M.F.; KASTELIC, J.P.; ADAMS, G.P.; COOK, B.; OLSON, W.O.; MAPLETOFT, R.J. The use of progestin in regimens for fixed-time artificial insemination in beef cattle. Theriogenology. v. 57, p. 1049-1059, 2002.

MARTINEZ, M.F.; KASTELIC, J.P.; BO, G.A. Effects of estradiol and some of its esters on gonadotrophin release and ovarian follicular dynamics in CIDR-treated beef cattle. Animal Reproduction Science, v. 86, p.37-52, 2005.

PALHAO, M.P.; BEG, M.A.; RODRIGUES, M.T.; GINTHER, O.J. Follicle and hormone dynamics in single versus double ovulating heifers. Reproduction. v. 138, p. 561-570, 2009.

PALHAO, M.P.; BEG, M.A.; RODRIGUES,; M.T.; GINTHER, O.J. Role of increased estradiol on altering the follicle diameters and gonadotropin concentrations that have been reported for doubleovulating heifers. Animal Reproduction Science. 122, p. 335-341, 2010.

SIDDIQUI, M.A.R.; GASTAL, E.L.; GASTAL, M.O.; ALMAMUN, M.; BEG, M.A.; GINTHER, O.J. Relationship of vascular perfusion of the wall of the preovulatory follicle in vitro fertilization and embryo development in heifers. Reproduction, v. 137, p. 689-697, 2009. 